\title{
Myelin Measurement Using Quantitative Magnetic Resonance Imaging: A Correlation Study Comparing Various Imaging Techniques in Patients with Multiple Sclerosis
}

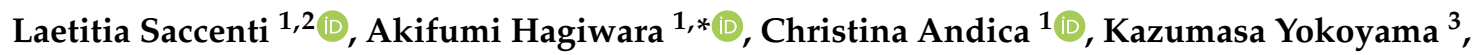 \\ Shohei Fujita ${ }^{1,4}$, Shimpei Kato ${ }^{1,4}$, Tomoko Maekawa ${ }^{1,4}$, Koji Kamagata ${ }^{1}$ (D), Alice Le Berre ${ }^{1,2}$, \\ Masaaki Hori ${ }^{1,5}$, Akihiko Wada ${ }^{1}$, Ukihide Tateishi ${ }^{6}$, Nobutaka Hattori ${ }^{3}$ (D) and Shigeki Aoki ${ }^{1}$ \\ 1 Department of Radiology, Juntendo University School of Medicine, 2-1-1, Hongo, Bunkyo-ku, \\ Tokyo 113-8421, Japan; laetitia.saccenti@gmail.com (L.S.); christina@juntendo.ac.jp (C.A.); \\ sh-fujita@juntendo.ac.jp (S.F.); s.kato.cy@juntendo.ac.jp (S.K.); t-maekawa@juntendo.ac.jp (T.M.); \\ kkamagat@juntendo.ac.jp (K.K.); alice.leberre@ymail.com (A.L.B.); mahori@juntendo.ac.jp (M.H.); \\ a-wada@juntendo.ac.jp (A.W.); saoki@juntendo.ac.jp (S.A.) \\ 2 Department of Radiology, St Joseph Hospital, 185 Rue Raymond Losserand, 75014 Paris, France \\ 3 Department of Neurology, Juntendo University School of Medicine, 2-1-1, Hongo, Bunkyo-ku, \\ Tokyo 113-8421, Japan; kazumasa@juntendo.ac.jp (K.Y.); nhattori@juntendo.ac.jp (N.H.) \\ 4 Department of Radiology, The University of Tokyo Graduate School of Medicine, 7-3-1, Hongo, Bunkyo-ku, \\ Tokyo 113-0033, Japan \\ 5 Department of Radiology, Toho University Omori Medical Center, 6-11-1, Omorinishi, Ota-ku, \\ Tokyo 143-8540, Japan \\ 6 Department of Diagnostic Radiology and Nuclear Medicine, Tokyo Medical and Dental University, 1-5-45, \\ Yushima, Bunkyo-ku, Tokyo 113-8519, Japan; ttisdrnm@tmd.ac.jp \\ * Correspondence: a-hagiwara@juntendo.ac.jp; Tel.: +81-3-3813-3111
}

Received: 3 January 2020; Accepted: 6 February 2020; Published: 8 February 2020

check for updates

\begin{abstract}
Evaluation of myelin by magnetic resonance imaging (MRI) is a difficult challenge, but holds promise in demyelinating diseases, such as multiple sclerosis (MS). Although multiple techniques have been developed, no gold standard has been established. This study aims to evaluate the correlation between synthetic MRI myelin volume fraction (SyMRI $\mathrm{MVF}_{\text {) }}$ ) and myelin fraction estimated by other techniques, i.e., magnetization transfer saturation (MTsat), T1-weighted images divided by T2-weighted images (T1w/T2w), and radial diffusivity (RD) in patients with MS. We also compared the sensitivities of these techniques for detecting MS-related myelin damage. SyMRI $\mathrm{MVF}_{\text {, }}$ MTsat, $\mathrm{T} 1 \mathrm{w} / \mathrm{T} 2 \mathrm{w}$, and RD were averaged on plaque, periplaque white matter, and normal-appearing white matter (NAWM). Pairwise correlation was calculated using Spearman's correlation analysis. For all segmented regions, strong correlations were found between SyMRI $\mathrm{MVF}_{\text {and }} \mathrm{T} 1 \mathrm{w} / \mathrm{T} 2 \mathrm{w}(\mathrm{Rho}=0.89)$, MTsat $(\mathrm{Rho}=0.82)$, or $\mathrm{RD}(\mathrm{Rho}=-0.75)$. For each technique, the average estimated myelin differed significantly among regions, but the percentage change of NAWM from both periplaque white matter and plaque were highest in SyMRI $\mathrm{IVF}_{\text {. SyMRI }}$. patients, with relevant results as compared to other well-studied techniques. Moreover, it presented better sensitivity for the detection of the difference between plaque or periplaque white matter and NAWM.
\end{abstract}

Keywords: myelin; multiple sclerosis; synthetic magnetic resonance imaging 


\section{Introduction}

Myelin is important in the transmission of neural information. It maintains the integrity of neural fibers and enhances the speed of propagation of action potentials, which are both essential for proper brain function [1,2]. Various magnetic resonance (MR) techniques have been used to study the pathological evolution of demyelinating diseases, such as multiple sclerosis (MS) [3]. Myelin imaging can potentially play an important role in the clinic, for predicting and monitoring responses to treatment: myelin status has been shown to be associated with changes in functional mobility following therapeutic exercises in patients with MS [4]. However, myelin assessment is a difficult challenge, as it cannot be seen directly on MRI, which has a millimeter-scale resolution. There is currently no recognized gold standard for myelin estimation, although myelin water fraction is one of the best validated and most commonly used quantitative measures for noninvasive assessment of myelin content in the brain [5]. To validate a new myelin estimation technique, histological studies are powerful and considered as the standard criterion, but its significance is somewhat limited as histological staining is evaluated based on optical density contrasts and therefore does not directly quantify myelin [6].

Recently, myelin measurement using quantitative synthetic MRI (SyMRI) was proposed [7]. This approach was originally developed for the simultaneous measurement of longitudinal T1 relaxation rate (R1), transverse $\mathrm{T} 2$ relaxation rate (R2), proton density (PD), and local radiofrequency field B1, using multislice, multiecho, and multidelay acquisition. The estimated B1 field is used for the correction of local variations in flip angle. Full head coverage is obtained in approximately 6 minutes [7]. From these absolute parameters, it is possible to create any contrast-weighted image that is clinically useful, including T1-weighted (T1w) or T2-weighted (T2w) images, using SyMRI software [8-10]. Using the same absolute parameters, SyMRI also allows myelin measurement [7]. Brain segmentation is based on pre-defined tissue characteristics. The myelin model assumes four compartments in the brain: myelin volume fraction (MVF), cellular volume fraction, free water fraction and excess parenchymal water volume fraction. The model postulates that each compartment has its own R1, R2, and PD, which contribute to the effective R1, R2, and PD of a specific voxel while exchanging magnetization with other partial volume compartments [7]. The MVF measured by SyMRI (SyMRI MVF) has been shown to correlate well with histological specimens in nonpathological brains [11] and brains with MS [12] and other myelin measurement methods in healthy subjects [13] and to show good repeatability [14,15] and reproducibility across different in-plane resolutions [16] and scanners [17]. Its clinical utility has been investigated in diseases such as MS [18,19], Sturge-Weber syndrome [20,21], and CADASIL [22].

A widely used myelin imaging method is based on magnetization transfer (MT), which is a physical process by which macromolecules and their closely associated water molecules cross-relax with protons in the free water pool. Based on this phenomenon, it is possible to quantify the protons bound to large molecules, which are not directly visible in MR images due to their extremely short $\mathrm{T} 2$. Radiofrequency pulse, applied at an offset to the resonance frequency of water, will cause saturation of the protons in the bound pool, but not those in free water. While returning to equilibrium, macromolecules exchange magnetization with free water, creating a measurable attenuation of the water signal. Myelin estimation using this technique is based on the assumption that most of the macromolecular content in the central nervous system forms part of myelin. Based on this theory, the MT ratio (MTR) has been widely used and has been shown to correlate well with histological myelin content [23]; moreover, an inverse correlation between clinical disability and average lesion MTR has also been reported [24]. However, MTR also correlates with R1, and thus water content, in the MS brain, indicating that inflammation and edema also influence MTR $[25,26]$. MT saturation (MTsat) imaging was developed to improve the MTR by decoupling the MTR from R1 [27]. MTsat shows higher contrast than MTR in the brain [27] and has been shown to correlate better than MTR with disability metrics in patients with MS [28]. On the other hand, quantitative MT imaging is time-consuming and its post-processing remains challenging. 
The T1w/T2w ratio map, which is calculated by dividing T1w images by $\mathrm{T} 2 \mathrm{w}$ images, is another approach for assessing tissue microstructure and indirectly, myelin content. T1 is shorter if the water environment contains more microstructural features, such as myelin and macromolecules. T2 is shorter if spins are in a geometrically restricted environment. Thus, the ratio of $\mathrm{T} 1 \mathrm{w} / \mathrm{T} 2 \mathrm{w}$ images is assumed to accentuate the intrinsic contrast of myelin. Although the T1w/T2w ratio is not a direct index of myelin, it is nevertheless considered a surrogate marker of myelin content [29]. This method was initially developed to evaluate intra-cortical myelin [29-31], and myelination of white matter in neonatal brains has also been investigated using this method [32]. The test-retest reliability of the T1w/T2w ratio has been reported to be high [33] and a histological study of patients with MS has shown that the T1w/T2w ratio in the cortex differed significantly between early-stage MS and healthy controls [34]. The major advantage of this method is the use of common T1w and T2w images that have already been acquired in the clinical routine. The T1w/T2w map provides an interesting assessment of tissue microstructure, despite not being highly specific to myelin [33].

On the other hand, diffusion tensor imaging, by measuring the microscopic movement of water in tissues, provides information on central nervous system tissue integrity and structure. Radial diffusivity (RD) provides information about water movement perpendicular to axonal tracts and is known to be related more to myelin integrity than axonal integrity [35]. In the corpus callosum of mouse brains, Song et al. [36] showed that the extent of increased RD reflects the severity of demyelination in the cuprizone demyelination mouse model, while RD decreases with the progression of remyelination. A pathological study in MS subjects concluded that increased RD correlates with demyelination, but also with axon injury [37]. However, diffusion tensor imaging is known to be affected not only by myelin density but also by fiber coherence [38].

A previous study by Hagiwara et al. [13] evaluated the correlation between SyMRI $\mathrm{MVF}, \mathrm{MTsat}$ and $\mathrm{T} 1 \mathrm{w} / \mathrm{T} 2 \mathrm{w}$ in healthy controls. There was a strong correlation in the WM between SyMRI $\mathrm{MVF}_{\text {and }}$ MTsat, indicating that both methods are similarly suitable for measuring myelin in the WM. Although MTsat, $\mathrm{T} 1 \mathrm{w} / \mathrm{T} 2 \mathrm{w}$, and RD are considered valid approaches for assessing microstructural integrity and hence myelin status, the relationship between these metrics has been largely unexplored [39]. Furthermore, the correlation between these techniques and a relatively new measure, SyMRI MVF, in MS patients has not been evaluated to date.

Correlation studies can reinforce the validity of a new myelin measurement technique if good correlations are found with well-studied methods. Therefore, the aim of our study was to evaluate the correlation between different methods used for myelin evaluation in patients with MS. We focused on four representative myelin imaging techniques, namely, SyMRI $\mathrm{MVF}_{\text {, }}$ MTsat, the T1w/T2w ratio, and RD. We also compared the sensitivities of these techniques for detecting MS-related myelin damage.

\section{Materials and Methods}

\subsection{Study Participants}

Thirty-seven patients with relapsing-remitting MS were prospectively included in this study, from March 2017 to July 2018. These patients were diagnosed with MS according to the standard McDonald criteria [40]. Of these patients, 14 did not have any plaque or had only small ( $<5 \mathrm{~mm}$ maximal length) plaques on brain MRI and 2 had diffuse extensive WM abnormalities. Therefore, these 16 patients were excluded and the remaining 21 patients were included in the analysis. Disability was assessed using the Expanded Disability Status Scale (EDSS) score. The demographic and clinical data of the patients with MS are provided in Table 1. The institutional review board of Juntendo University Hospital approved this study on 18 March 2016 and written informed consent was obtained from all participants. The ethical approval number is 15-212. 
Table 1. Demographic and clinical details of study participants.

\begin{tabular}{cc}
\hline & MS Patients \\
\hline No. of Subjects & 21 \\
\hline Mean Age (yr) & $37.9 \pm 9.9$ \\
\hline Sex (Male/Female) & $2: 19$ \\
\hline Disease Duration (Mean) (yr) & $8.7 \pm 6.5$ \\
\hline EDSS Score (range) & $1(0-2)$ \\
\hline
\end{tabular}

\subsection{MRI Acquisition Protocol}

A 3-T MR system (MAGNETOM Prisma, Siemens Healthcare, Erlangen, Germany) with a 64-channel head coil was used for all imaging. All patients underwent MR relaxometry, MTsat determination, and diffusion-weighted imaging.

\subsection{Acquisition and Processing of SyMRI Data}

MR relaxometry was performed with the QRAPMASTER (quantification of relaxation times and proton density by multi-echo acquisition of a saturation recovery using turbo spin-echo readout) pulse sequence, which is a multi-slice, multi-echo, and multi-saturation delay acquisition sequence. Two sets of echo times (TE) and 4 sets of delay times were used to generate 8 complex images in each section in order to quantify R1, R2, and PD. The TE were 22 and $99 \mathrm{~ms}$, the delay times were 170, 620, 1970 , and $4220 \mathrm{~ms}$ and the repetition time (TR) was $4250 \mathrm{~ms}$. An in-plane resolution of $0.8 \times 0.8 \mathrm{~mm}$ with a slice thickness/gap of 4.0/1.0 mm was used for 30 slices. The field-of-view (FOV) was set to $230 \times 186 \mathrm{~mm}$ with a matrix size of $320 \times 208$. The acquisition time was $5 \mathrm{~min}$ and $8 \mathrm{~s}$.

With the assumption that all the R1, R2, and PD values of MVF, excess parenchymal water volume fraction, cellular volume fraction, and free water volume fraction contribute to the effective R1, R2, and PD in each acquisition voxel; a model was produced to estimate partial volumes of these four compartments, as described by Warntjes et al. [7]. This was done by running Bloch equations and optimizing model parameters in a spatially normalized and averaged brain from a group of healthy controls. Damage to myelin, even in the NAWM of patients with MS [18], is supposed to cause deviation from the R1, R2, and PD in the healthy state, leading to a decrease in the calculated

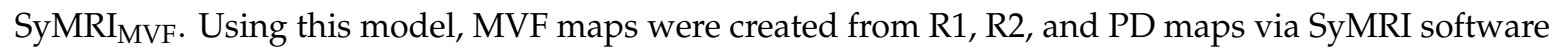
(version 8.04; SyntheticMR, Linköping, Sweden).

Even though other myelin imaging techniques require scaling factors for estimating MVF from the measured macromolecular pool or myelin water, this procedure was not necessary because SyMRI $\mathrm{MVF}_{\mathrm{F}}$ directly estimates the volume fraction of myelin in each voxel [7].

\subsection{Processing of the $T 1 w / T 2 w$ Ratio}

Synthetic T1w and T2w images were created using post-processing TR of $500 \mathrm{~ms}$ and TE of $10 \mathrm{~ms}$ and TR of $4500 \mathrm{~ms}$ and TE of $100 \mathrm{~ms}$, respectively, on SyMRI software based on R1, R2, and PD maps. To reduce the effect of intensity scaling, we used a calibration algorithm based on 2 anatomical masks (eye and temporal muscle), as proposed by Ganzetti et al. [41]. We recorded the modes as reference values for the eyes as follows: 28.2 for T1w images and 99.9 for T2w images. For the temporal muscle, these values were 58.6 for T1w images and 21.1 for T2w images. After calibrating the T1w and T2w images, their ratio was calculated to produce $\mathrm{T} 1 \mathrm{w} / \mathrm{T} 2 \mathrm{w}$ ratio images. The $\mathrm{T} 1 \mathrm{w} / \mathrm{T} 2 \mathrm{w}$ ratio was further linearly calibrated, as reported in a previous study [13], to be comparable to SyMRI MVF.

\subsection{Acquisition and Processing of MTsat}

Three three-dimensional (3D) multi-echo fast low-angle shot (FLASH) sequences were run with predominant T1-, PD-, and MT-weighting for all subjects. For T1w images, TR/excitation flip angle 
$\alpha=10 \mathrm{~ms} / 13^{\circ}$ was used; for PD- and MT-weighted images, $24 \mathrm{~ms} / 4^{\circ}$ was used. For MT-weighted images, excitation was preceded by an off-resonance Gaussian-shaped RF pulse (frequency offset from water resonance, $1.2 \mathrm{kHz}$; pulse duration, $9.984 \mathrm{~ms}$; and nominal flip angle, $500^{\circ}$ ). Other imaging parameters were: slice thickness, $1.8 \mathrm{~mm}$; 104 slices; FOV $224 \times 224 \mathrm{~mm}$; matrix $128 \times 128$, parallel imaging using GRAPPA factor 2 in the phase-encoding direction; 7/8 partial Fourier acquisition in the partition direction; bandwidth $260 \mathrm{~Hz} /$ pixel; and total acquisition time of $6 \mathrm{~min}$ and $25 \mathrm{~s}$. These 3 images were used to calculate the MTsat index, as described by Helms et al. [27]. The MTsat index was also further linearly calibrated as reported previously [13].

\subsection{Acquisition and Processing of Radial Diffusivity}

Whole-brain diffusion-weighted imaging was performed using spin-echo planar imaging employing a $b$-value of $1000 \mathrm{~s} / \mathrm{mm}^{2}$ along 64 uniformly distributed motion-probing directions in the anteroposterior phase-encoding direction. Standard and reverse phase-encoded blipped images with no diffusion weighting $\left(b=0 \mathrm{~s} / \mathrm{mm}^{2}\right)$ were also acquired. Other imaging parameters were TR, $3300 \mathrm{~ms}$; TE, $70 \mathrm{~ms}$; flip angle, $90^{\circ}$; FOV, $229 \times 229 \mathrm{~mm}$; matrix size, $130 \times 130$; resolution, $1.8 \times 1.8 \mathrm{~mm}$; slice thickness, $1.6 \mathrm{~mm}$; acquisition time, $3 \mathrm{~min}$ and $55 \mathrm{~s}$. All datasets were free from severe artifacts, such as gross geometric distortion, signal dropout, or bulk motion. Diffusion-weighted imaging data were then corrected for susceptibility-induced geometric distortions, eddy current distortions, and inter-volume subject motion using TOPUP and EDDY tools [42]. A single-tensor RD map was generated using the DTIFIT tool on FSL software v5.0.11. Linear transformation was performed to register the acquired diffusion and MTsat images to SyMRI images using BBR and FLIRT, respectively, implemented in FSL.

\subsection{Image Analysis}

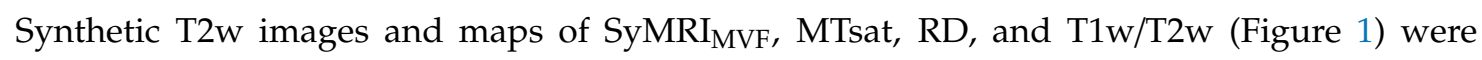
analyzed by using ITK-SNAP software (3.8.0-beta version) by a radiologist (L.S.) with 3 years of experience. Plaques were defined as a WM area of more than $5 \mathrm{~mm}$ in diameter in the supratentorial area, with abnormally high intensity on $\mathrm{T} 2 \mathrm{w}$ images. Plaques were semi-automatically segmented on synthetic T2w images using an adaptive brush tool for automatic and objective adjustment to lesion boundaries, choosing an adaptive algorithm with high granularity and low smoothness to undersegment plaques slightly in order to mitigate possible misregistration and the partial volume effect. Periplaque regions were manually segmented with a small round-shaped brush (approximately $1 / 5$ th of plaque diameter). Periplaque was defined as a visually normal-appearing WM (NAWM) area on a synthetic T2w image closest to a plaque $[19,43,44]$. Around each plaque, up to 6 areas were added in order to encircle the plaque and, together, these were considered as a periplaque region-of-interest (ROI). NAWM was manually segmented with a round-shaped brush on the WM contralateral to each plaque while avoiding other plaques. All the ROIs placed on synthetic T2w images were copied and pasted onto the SyMRI $\mathrm{MVF}_{\text {, }}$ T1w/T2w, MTsat, and RD maps.

\subsection{Statistical Analysis}

For acquired myelin-sensitive metrics, normality was tested with the Shapiro-Wilk test. Because not all data were normally distributed, we used the Steel-Dwass nonparametric test for multiple comparisons to compare ROI volumes and the values of SyMRI $\mathrm{MVF}_{\mathrm{F}}, \mathrm{T} 1 \mathrm{w} / \mathrm{T} 2 \mathrm{w}, \mathrm{MTsat}$, and RD among plaque, periplaque, and NAWM tissue. The percentage changes in plaque or periplaque relative to NAWM were also calculated and compared among different metrics using the Steel-Dwass test. The signs of negative percentage changes were inverted before comparison. 


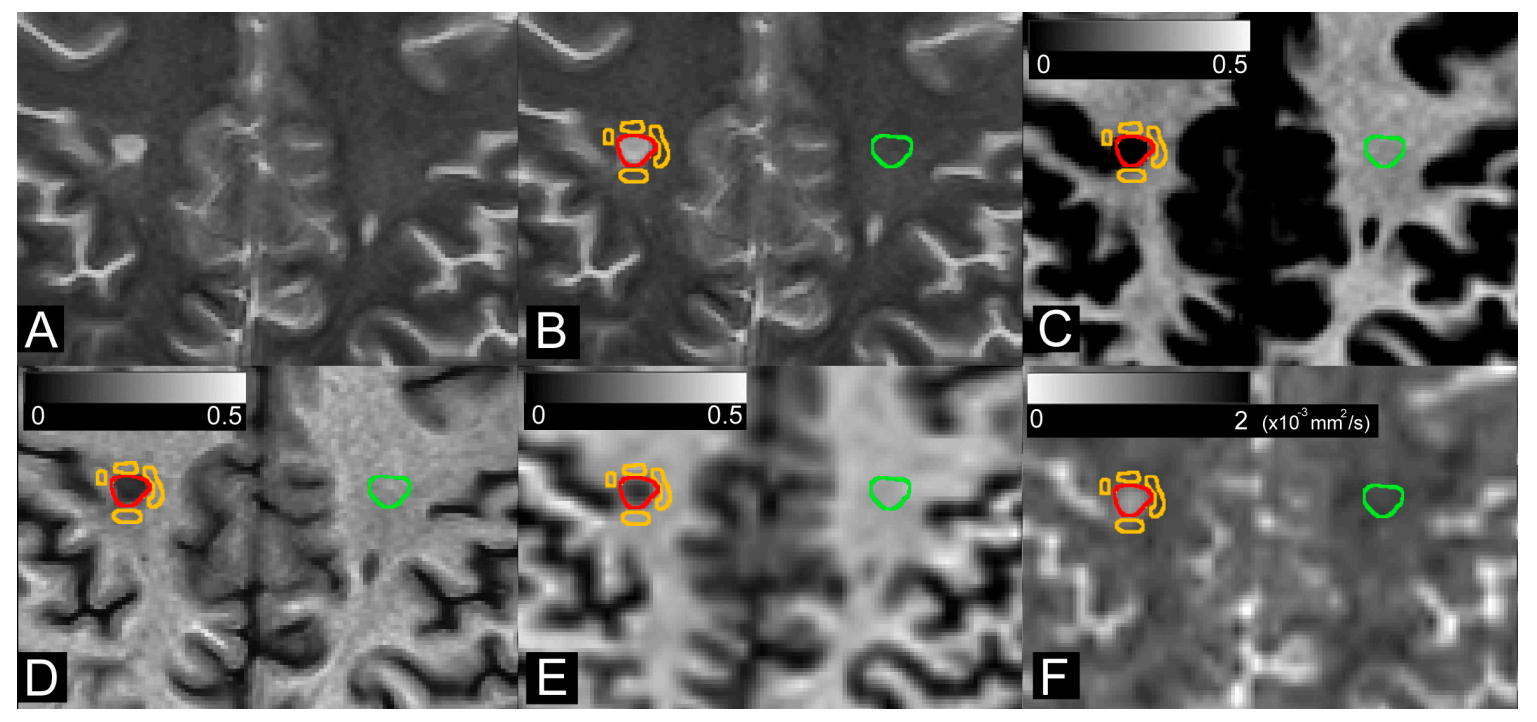

Figure 1. Synthetic T2-weighted images (A) were used for semi-automated segmentation of plaque (red), periplaque (orange), and contralateral normal-appearing white matter (green) (B). Regions-of-interest were copied and pasted onto SyMRI $\mathrm{MVF}_{\mathbf{H}}(\mathbf{C}), \mathrm{T} 1 \mathrm{w} / \mathrm{T} 2 \mathrm{w}(\mathbf{D})$, MTsat (E), and RD (F) maps.

Spearman's rank order correlation analysis was used to investigate the correlation among myelin-sensitive metrics for plaque, periplaque, NAWM, and all regions. The signs of negative coefficients were inverted for further analysis and classification. Correlation coefficients across metrics were compared in plaque, periplaque, NAWM, and overall regions using a percentile bootstrap method, as described by Wilcox [45,46]. EDSS and disease duration were also correlated with myelin-sensitive metrics in the plaque, periplaque, and NAWM regions, averaged in each patient via Spearman's rank order correlation analysis. Then, $p$-values for correlation coefficients and their comparisons were controlled for the false discovery rate [47]. Spearman's $\rho$ correlation coefficients were classified as follows: $0-0.30$, very weak; $0.30-0.50$, weak; $0.50-0.70$, moderate; $0.70-0.90$, strong; and 0.90-1.00, very strong. A 2 -sided $p$-value $<.05$ was considered significant. Shapiro-Wilk and Steel-Dwass tests were performed with the software package $\mathrm{R}$, version 3.3.3. Calculation of correlation coefficients and comparisons of these were performed on Matlab (release R2015b, MathWorks, Natick, Massachusetts, United States).

\section{Results}

Ninety-two isolated plaques were segmented; thus, the total number of ROIs was 276, including plaque, periplaque, and NAWM. The median number of plaques per patient was 5 (range, 1-7). The mean ( \pm standard deviation) size of plaque, periplaque, and NAWM ROIs were $144 \pm 111,144 \pm$ 112 , and $145 \pm 113 \mathrm{~mm}^{3}$, respectively. There were no significant differences between the volumes of these ROIs $(p>0.99)$.

For each myelin measurement technique, metrics were significantly different among plaque, periplaque, and NAWM regions (Figure 2), while the percentage changes between NAWM and both periplaque and plaque regions were significantly the highest for SyMRI $\mathrm{MVF}_{\text {(Figure 3). }}$

All segmented regions together, there were strong significant correlations between $S_{Y M R I} \mathrm{MVF}_{\text {, }}$ $\mathrm{T} 1 \mathrm{w} / \mathrm{T} 2 \mathrm{w}, \mathrm{MTsat}$, and RD, except between RD and T1w/T2w which presented moderate significant

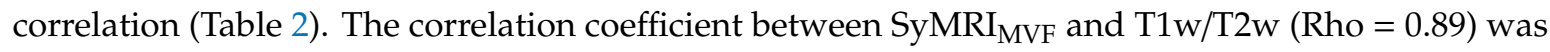
significantly higher than all other correlations (Table S1). Correlation coefficients involving RD were significantly lower than other correlations. 


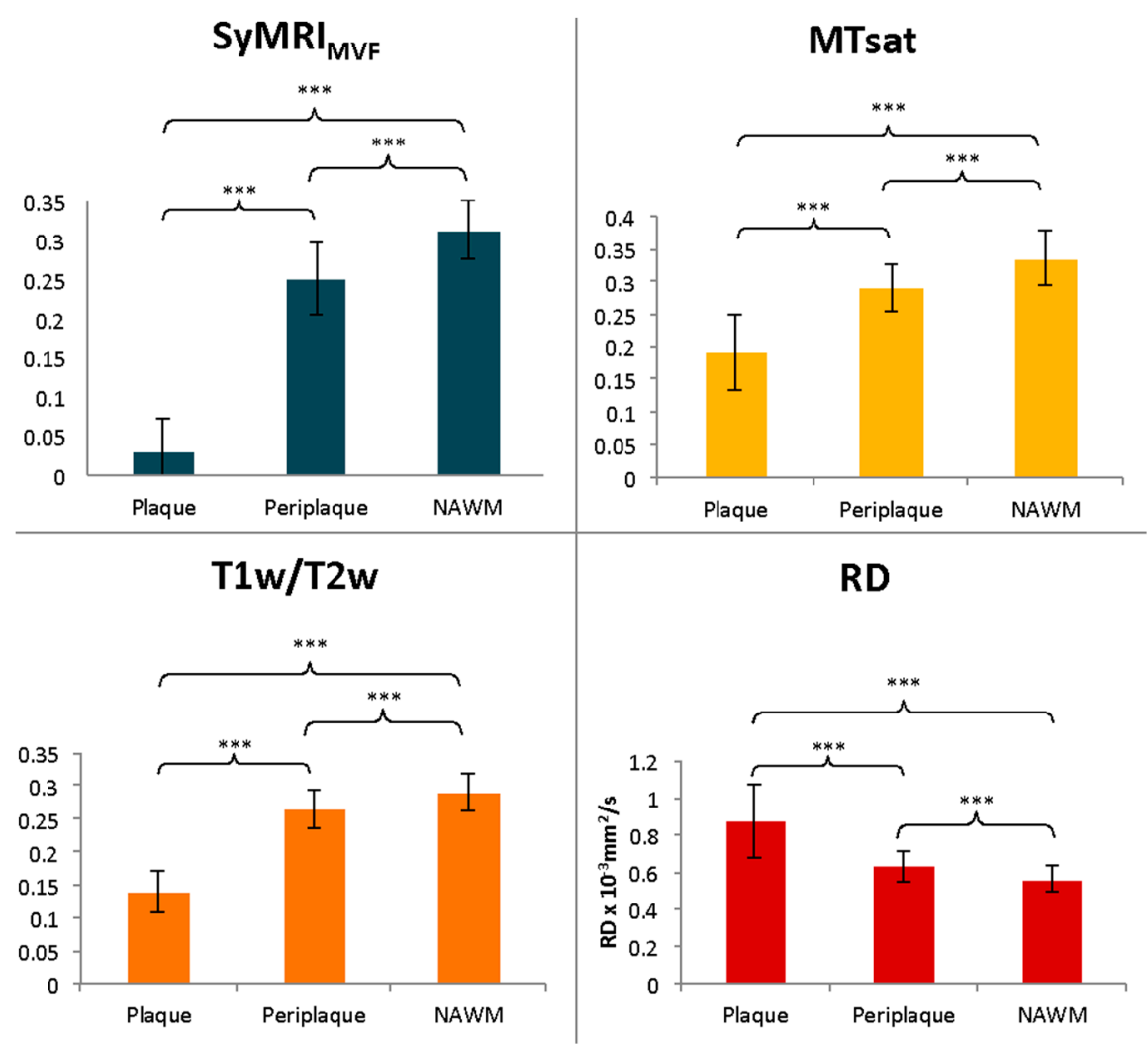

Figure 2. Estimation of myelin with 4 different techniques (SyMRI ${ }_{M V F}, M T s a t, T 1 w / T 2 w$, and RD). Each technique can differentiate plaque, periplaque, and NAWM regions. These metrics were the lowest in plaque for SyMRI $\mathrm{MVF}_{\mathrm{F}}$, MTsat, and T1w/T2w and the highest in plaque for RD. ${ }^{* * *} p<0.001$.

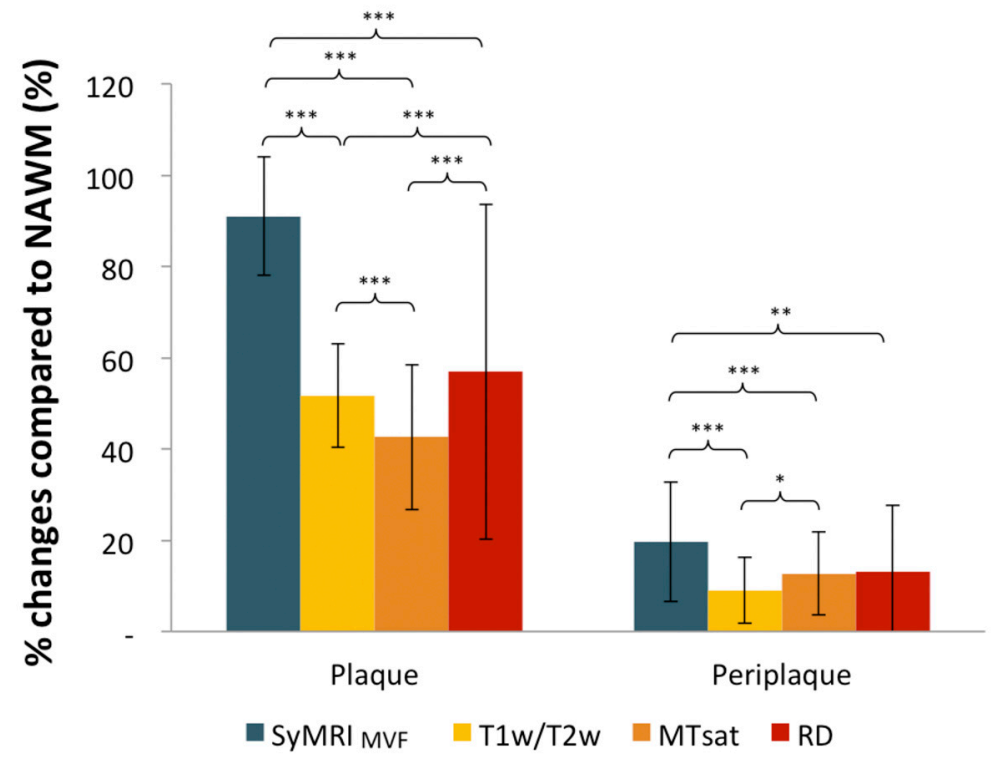

Figure 3. Percentage changes in the metrics (SyMRI $I_{M V F}, T 1 w / T 2 w, M T s a t$, and RD) for plaque and periplaque compared to NAWM regions. SyMRI MVF showed the highest contrast between NAWM and both plaque and periplaque regions. ${ }^{*} p<0.05 .{ }^{* *} p<0.01$. ${ }^{* *} p<0.001$. 
Table 2. Correlation matrix of Spearman's rank order correlation analysis.

\begin{tabular}{cccc}
\hline & MTsat & T1w/T2w & RD \\
\hline SyMRI $_{\text {MVF }}$ & $0.82[0.77-0.87]^{* * *}$ & $0.89[0.85-0.92]^{* * *}$ & $-0.75[-0.80-(-0.69)]^{* * *}$ \\
MTsat & $0.80[0.74-0.85]^{* * *}$ & $-0.72[-0.78-(-0.65)]^{* * *}$ \\
T1w/T2w & & $-0.66[-0.73-(-0.57)]^{* * *}$ \\
\hline Data are the Spearman's Rho correlation coefficients \pm 95\% confidence interval. For the segmented regions overall, \\
strong correlations were found between SyMRI ${ }_{\text {MVF }}^{*}$, T1w/T2w, MTsat, and RD, except for RD and T1w/T2w, \\
which were moderately correlated. All correlations were statistically significant. ${ }^{* * * *} p<0.001$.
\end{tabular}

We also evaluated the correlation in the subgroups of segmented ROIs (Figure 4, Table 3). Moderate to strong, statistically significant correlations between SyMRI $\mathrm{MVF}_{\mathrm{F}}$, MTsat, and T1w/T2w were found in plaque subgroup, while correlations between RD and other metrics were weak to moderate. Weak to moderate statistically significant correlations between all metrics were found in the periplaque subgroup, except between $\mathrm{T} 1 \mathrm{w} / \mathrm{T} 2 \mathrm{w}$ and $\mathrm{RD}$, which were nonsignificant. Very weak to weak significant correlations between SyMRI $\mathrm{MVF}, \mathrm{T} 1 \mathrm{w} / \mathrm{T} 2 \mathrm{w}$, and MTsat were detected in NAWM regions. There was no significant correlation between RD and other metrics in the NAWM subgroup. Overall, stronger correlations were found in the plaque subgroup than in the periplaque and NAWM subgroups. Furthermore, correlation coefficients involving RD were lower than other correlations in subgroup analysis (Table S1).
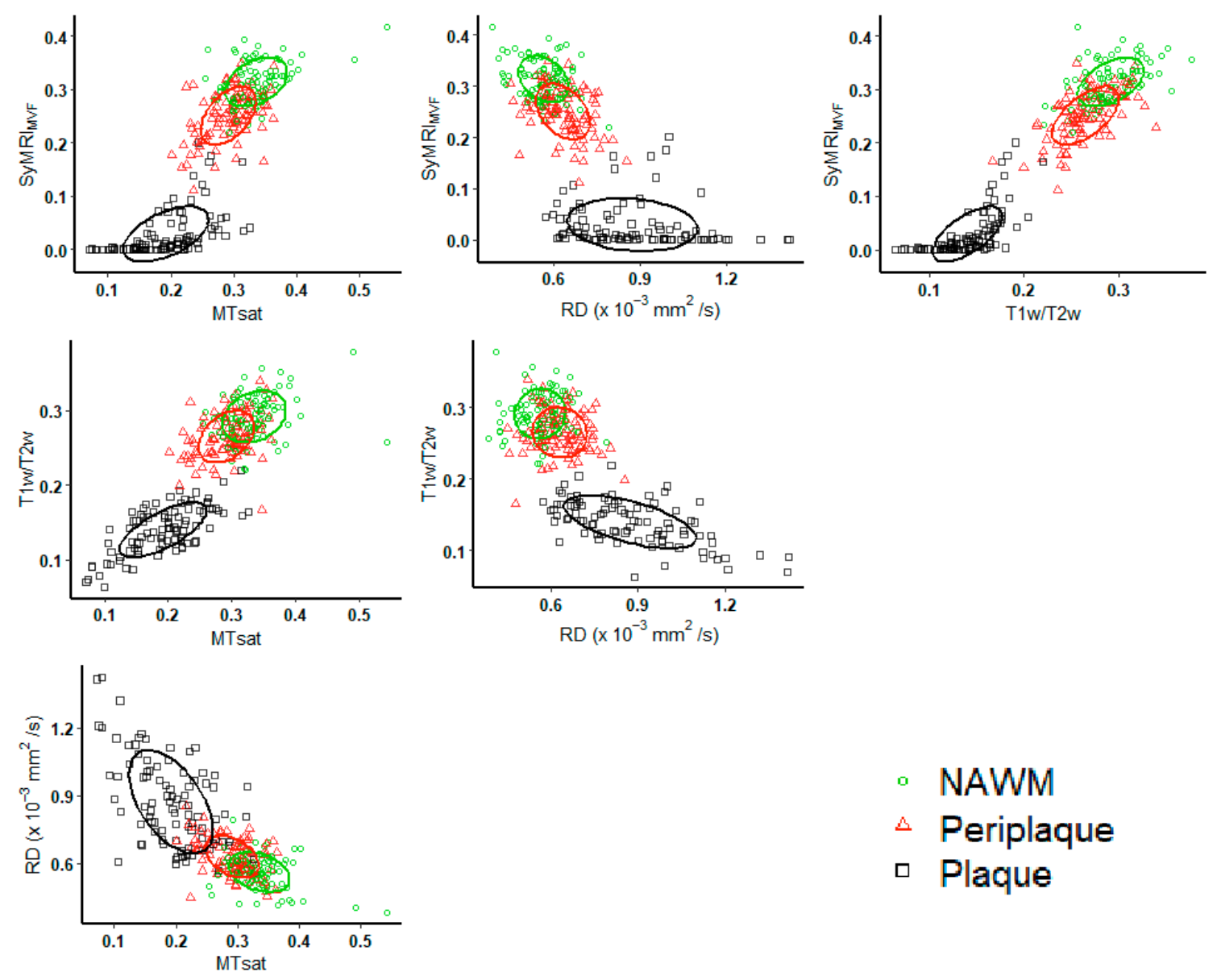

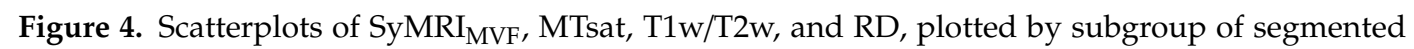
regions-of-interest: black square $=$ plaque, red triangle $=$ periplaque, green circle $=\mathrm{NAWM}$. Plot concentration ellipses are also drawn, with concentration levels of 0.5 . 
Table 3. Correlation among SyMRI ${ }_{\mathrm{MVF}}, \mathrm{MTsat}, \mathrm{T} 1 \mathrm{w} / \mathrm{T} 2 \mathrm{w}$, and $\mathrm{RD}$ for plaque, periplaque, and NAWM subgroups.

\begin{tabular}{|c|c|c|c|c|}
\hline & & MTsat & $\mathrm{T} 1 \mathrm{w} / \mathrm{T} 2 \mathrm{w}$ & RD \\
\hline Plaque & $\begin{array}{c}\text { SyMRI }_{M V F} \\
\text { MTsat } \\
\text { T1w/T2w }\end{array}$ & $0.70[0.58-0.79]^{* * *}$ & $\left.\begin{array}{l}0.78[0.67-0.86] \\
0.64[0.47-0.77]\end{array}\right]^{* * *}$ & $\begin{array}{l}-0.38[-0.53-(-0.19)]^{* * *} \\
-0.48[-0.65-(-0.29)]^{* * *} \\
-0.58[-0.71-(-0.41)]^{* * *}\end{array}$ \\
\hline Periplaque & $\begin{array}{c}\text { SyMRI }_{M V F} \\
\text { MTsat } \\
\text { T1w/T2w }\end{array}$ & $0.45[0.23-0.60]^{* * *}$ & $\begin{array}{l}0.62[0.49-0.74]^{* * *} \\
0.41[0.22-0.59]^{* * *}\end{array}$ & $\begin{array}{l}0.41[0.23-0.56]^{* * *} \\
0.40[0.19-0.59]^{* * *} \\
-0.09[-0.31-0.13]\end{array}$ \\
\hline NAWM & $\begin{array}{c}\text { SyMRI }_{M V F} \\
\text { MTsat } \\
\text { T1w/T2w }\end{array}$ & $0.33[0.13-0.51]^{* *}$ & $\begin{array}{c}0.50[0.32-0.67] \\
0.28[0.11-0.47]^{* *}\end{array}$ & $\begin{array}{c}-0.21[-0.41-(-0.02)] \\
-0.20[-0.45-0.02] \\
0.11[-0.10-0.29]\end{array}$ \\
\hline
\end{tabular}

Data are the Spearman's Rho correlation coefficients $\pm 95 \%$ confidence interval. ${ }^{*} p<0.05,{ }^{* *} p<0.01,{ }^{* * *} p<0.001$.

Disease duration was significantly correlated with SyMRI $\mathrm{MVF}_{\text {F }}$ of NAWM regions averaged in each patient $($ Rho $=-0.63, p=0.002)$ and with RD of NAWM regions averaged in each patient $($ Rho $=0.51$, $p=0.02$; Table 4). On the other hand, no significant correlation was found between SyMRI $\mathrm{MVF}_{\mathrm{MF}}$ or RD and EDSS, and no significant correlation was found between NAWM MTsat or T1w/T2w and EDSS or disease duration. Likewise, no significant correlation was found between myelin estimation in plaque or periplaque and EDSS or disease duration, regardless of the myelin estimation technique used.

Table 4. Correlation between clinical scores and SyMRI $I_{M V F}$, MTsat, T1w/T2w, or RD in plaque, periplaque, and NAWM.

\begin{tabular}{|c|c|c|c|c|c|}
\hline & & SyMRI $_{\text {MVF }}$ & MTsat & $\mathrm{T} 1 \mathrm{w} / \mathrm{T} 2 \mathrm{w}$ & RD \\
\hline \multirow[t]{2}{*}{ Plaque } & EDSS & $-0.10[-0.57$ to 0.39$]$ & $-0.29[-0.71$ to 0.22$]$ & $-0.0014[-0.49$ to 0.48$]$ & $-0.12[-0.56$ to 0.36$]$ \\
\hline & Disease Duration & $0.20[-0.36$ to 0.64$]$ & $0.17[-0.34$ to 0.63$]$ & $-0.17[-0.67$ to 0.38$]$ & $0.32[-0.17$ to 0.77$]$ \\
\hline \multirow[t]{2}{*}{ Periplaque } & EDSS & $0.13[-0.33$ to 0.51$]$ & $0.12[-0.38$ to 0.57$]$ & $0.24[-0.26$ to 0.66$]$ & $-0.31[-0.68$ to 0.11$]$ \\
\hline & Disease Duration & $-0.00065[-0.52$ to 0.45$]$ & $-0.12[-0.51$ to 0.38$]$ & $-0.24[-0.67$ to 0.26$]$ & $0.23[-0.25$ to 0.66$]$ \\
\hline \multirow[t]{2}{*}{ NAWM } & EDSS & $0.45[0.031$ to 0.76$]$ & $0.15[-0.32$ to 0.60$]$ & $0.23[-0.28$ to 0.63$]$ & $-0.47[-0.80$ to -0.056$]$ \\
\hline & Disease Duration & $-0.63[-0.81 \text { to }-0.32]^{* *}$ & $-0.39[-0.70$ to 0.072$]$ & $-0.38[-0.72$ to 0.077$]$ & $0.51[0.11 \text { to } 0.81]^{*}$ \\
\hline
\end{tabular}

Data are the Spearman's Rho correlation coefficients $\pm 95 \%$ confidence interval. ${ }^{*} p<0.05,{ }^{* *} p<0.01$, and ${ }^{* * *} p<0.001$.

\section{Discussion}

In this study, we compared four techniques for measuring myelin in the WM of patients with MS. Among all segmented ROIs, we found strong correlations between SyMRI $\mathrm{IVF}_{\mathrm{MF}}$ and the other measures. Furthermore, in our study, the contrast for distinguishing plaque and periplaque from NAWM regions was higher with SyMRI MVF than with the other myelin imaging techniques. Because myelin debris is assumed to have much lower R2 than the tightly packed myelin, the contribution of myelin debris to SyMRI $\mathrm{MVF}_{\mathrm{MF}}$ is expected to be small. Other myelin imaging techniques may be sensitive to myelin debris or other molecules, which may have resulted in the observed lower contrast between plaque or periplaque and NAWM. Notably, gray matter to WM contrast in the SyMRI MVF (see Figure 1) was reported to be higher than MTsat and T1w/T2w in a previous study by Hagiwara et al. [13] and they concluded that the gray matter to WM contrast in SyMRI MVF was nearer to the results of previous histological studies compared with MTsat and T1w/T2w. Their results also indicate that MTsat and T1w/T2w may be sensitive to other molecules than myelin.

Our results are also in line with those of other previous studies. Hagiwara et al. [43] obtained better contrast using SyMRI MVF than R1, R2, and PD in the WM of MS patients. The MS disease process extends beyond the borders of visible plaques on conventional $\mathrm{T} 2 \mathrm{w}$ images, and periplaque and NAWM abnormalities are related to different histological processes, according to pathology studies [44]. Periplaque may be abnormal because of Wallerian degeneration and retrograde degeneration of the cell body [48]. Additionally, plaque evolution (i.e., the demyelination/remyelination process) may explain the difference in myelin measurements in the periplaque regions. In NAWM, microglial activation and axonal degeneration might be the main pathological underpinnings of subtle MRI abnormalities [49]. 
Because the SyMRI model defines myelin at predetermined R1, R2, and PD, based on a group of healthy control subjects [7], any disease process that changes myelin integrity, such as edema or partial demyelination, should theoretically affect SyMRI MVF. Hence, SyMRI MVF may be merely a potential additional metric to be confronted with others, such as diffusion tensor imaging and MT imaging. The higher contrast between plaque, periplaque, and NAWM regions seen with SyMRI $\mathrm{IVF}_{\mathrm{F}}$ than with other metrics may indicate that SyMRI MVF has better sensitivity to myelin than other techniques. The major advantage of SyMRI $\mathrm{MVF}_{\text {in }}$ is that the myelin estimation is fully automated and rapid (post-processing time of less than $1 \mathrm{~min}$ ) and the same 6-minute unique sequence acquisition makes it possible to obtain conventional contrast-weighted images used routinely in the clinic (T1w, T2w, and FLAIR) and other contrast-weighted images (double inversion recovery, phase-sensitive recovery images), with better contrast for MS plaques detection than found with conventional images [10].

However, subgroup analyses in this study found lower correlations between the techniques, especially in NAWM regions. Calculation of correlation coefficients depends largely on the range of the measured values: if the range is wide, the correlation will be higher than if the range is narrow [50]. Hence, it is natural that the correlation coefficient was lowest when calculated for only NAWM, which is expected to be homogeneous, as compared with pathological plaque and periplaque regions. These results were similar to those of previous myelin correlation studies: correlation between the myelin water fraction and MTR was very weak within NAWM in MS patients [51]. Another correlation study analyzed the relationship between RD, MTR, and the myelin water fraction and found good agreement when estimating WM microstructural damage in MS patients, with the lowest correlations found in the NAWM [39]. Recently, O'Muircheartaigh et al. [52] also showed strong correlations throughout the brain between the myelin water fraction, MTR, and quantitative T1, but these relationships varied in different tissue types. In NAWM, no significant correlation was found. All these studies concluded that the different quantitative techniques are at least partially correlated with each other but are sensitive to different aspects of the pathology and may provide complementary information about underlying brain tissues [53].

Although promising advances in imaging myelination have been reported, inflammation can interfere with measuring myelin and axons. NAWM in MS patients often exhibits chronic injury, microglial activation, gliosis, and increased expression of proteolytic enzymes [54]. Moll et al. [49], in a pathology-imaging correlation study, concluded that the pathological substrates of MTR changes in NAWM in MS patients could be attributed to axonal degeneration and microglial activation, but were not correlated to myelin staining.

Even though the specificity of myelin quantification by SyMRI $\mathrm{MVF}_{\mathrm{F}}$ was not proven in this study, SyMRI $_{\text {MVF }}$ averaged for the NAWM in each patient presented a significant correlation with disease duration. This emphasizes the clinical relevance of NAWM and the importance of detecting damage to this tissue, considering that the association between clinical findings and lesion volumes is reportedly poor [55].

No significant correlation was found between EDSS and the estimated myelin in the NAWM, but the range of EDSS in this study (i.e., $0-2$ ) was small. Further studies that include a larger population of patients with a wider range of disability scores are warranted.

Our study had some limitations. First, we did not compare SyMRI to myelin water imaging, which is one of the best validated techniques. Further studies comparing SyMRI MVF and myelin water imaging in patients with MS should be conducted to analyze the association between these metrics. Second, we chose to investigate MTsat, which is thought to be an improvement over MTR, but some new magnetization transfer techniques, for example, inhomogeneous magnetization transfer, have been developed that may increase the sensitivity and specificity for myelin [56]. Third, even though we slightly undersegmented plaques to mitigate possible misregistration and the partial volume effect, the acquired images were gapped at $1 \mathrm{~mm}$ and there may have been inevitable partial volume effects that have contributed to bias in the results. Fourth, we focused on T2w WM hyperintense lesional tissue without taking the T1w signal and contrast-enhancement, which requires the administration of 
a gadolinium-based contrast agent, into account when examining the activity of lesions. In the future, contrast-enhanced images could be used to separate subgroups or inflammatory lesions and study the differences in the metrics. A previous study by Blystad et al. [57] compared T1, T2, and PD measured by SyMRI between enhancing and NAWM and showed that enhancing lesions have higher T1, T2, and PD values than NAWM. Even though there has been no study that has evaluated the difference in SyMRI MVF between enhancing lesions and NAWM, it is highly likely that enhancing lesions show decreased SyMRI ${ }_{\text {MVF }}$ than NAWM. However, to what degree inflammation contributes to SyMRI MVF is still unclear and a future histological study on active MS lesions is awaited. Additionally, the patients included in the present study were all being followed-up for relapsing-remitting MS. In future studies, myelin should also be evaluated in patients with clinically isolated syndromes and primary/secondary progressive MS and the correlations between the techniques assessed.

Lastly, concerning NAWM, we did not take into account the location of the ROI; hence, future studies should include more patients and, using anatomical masks, evaluate subgroups or anatomical regions in order to establish the influence of ROI location on the metrics.

\section{Conclusions}

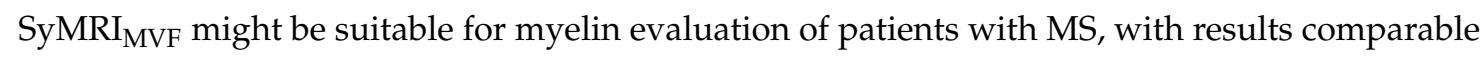
to those of other well-studied myelin evaluation techniques (MTsat, T1w/T2w, and RD). Moreover, it presented better sensitivity for detecting the difference between plaque or periplaque regions and NAWM, which warrants further investigation for SyMRI $\mathrm{MVF}_{\text {fo }}$ to useful for diagnosis and prognosis evaluation. Subregional analysis demonstrated a weaker but significant correlation in

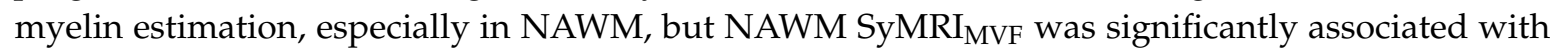
disease duration. SyMRI MVF could therefore yield additional complementary information about microstructural tissue damage in patients with MS.

Supplementary Materials: The following are available online at http://www.mdpi.com/2073-4409/9/2/393/s1, Table S1: $p$ values for comparisons of Spearman's rho correlation coefficients across SyMRI MVF, MTsat, T1w/T2w and RD. These $p$ values are corrected for false discovery rate.

Author Contributions: L.S., A.H., C.A., M.H., U.T. and S.A. contributed to the conception and design of the study. L.S., K.Y., S.F., S.K., T.M., K.K., A.L.B., A.W. and N.H. contributed to the data collection and acquisition and analysis of data. L.S., A.H., C.A. and S.A. contributed to drafting the text and preparing the figures. All authors have reviewed and approved the contents of the manuscript.

Funding: This work was supported by the budget for the promotion of strategic international standardization given by the Ministry of Economy, Industry and Trade (METI); JSPS KAKENHI grant number 19K17177, 19K17150, 18K15643 and JP16H06280; the Brain/MINDS program from the Japan Agency for Medical Research and Development (AMED) Grant Number 18dm0207016h0005, JP19dm0307024 and JP19dm0307101; a Grant-in-Aid for Special Research in subsidies for ordinary expenses of private schools from The Promotion and Mutual Aid Corporation for Private Schools of Japan; and Brain/MINDS program from the Japan Agency for Medical Research and Development (AMED) Grant Number JP19dm0307024 and JP19dm0307101.

Acknowledgments: We are grateful for the cooperation of the Japan Quantitative Imaging Biomarkers Alliance (J-QIBA) and Radiological Society of North America QIBA (RSNA-QIBA).

Conflicts of Interest: The authors declare no conflicts of interest.

\section{References}

1. de Hoz, L.; Simons, M. The emerging functions of oligodendrocytes in regulating neuronal network behaviour. BioEssays 2015, 37, 60-69. [CrossRef]

2. Duval, T.; Stikov, N.; Cohen-Adad, J. Modeling white matter microstructure. Funct. Neurol. 2016, 31, $217-228$. [CrossRef] [PubMed]

3. Heath, F.; Hurley, S.A.; Johansen-Berg, H.; Sampaio-Baptista, C. Advances in noninvasive myelin imaging. Dev. Neurobiol. 2018, 78, 136-151. [CrossRef] [PubMed]

4. King, E.; Sabatier, M.; Hoque, M.; Kesar, T.; Backus, D.; Borich, M. Myelin status is associated with change in functional mobility following slope walking in people with multiple sclerosis. Mult. Scler. J. Exp. Transl. Clin. 2018, 4. [CrossRef] [PubMed] 
5. Prasloski, T.; Rauscher, A.; MacKay, A.L.; Hodgson, M.; Vavasour, I.M.; Laule, C.; Mädler, B. Rapid whole cerebrum myelin water imaging using a 3D GRASE sequence. NeuroImage 2012, 63, 533-539. [CrossRef]

6. Stüber, C.; Morawski, M.; Schäfer, A.; Labadie, C.; Wähnert, M.; Leuze, C.; Streicher, M.; Barapatre, N.; Reimann, K.; Geyer, S.; et al. Myelin and iron concentration in the human brain: A quantitative study of MRI contrast. NeuroImage 2014, 93, 95-106. [CrossRef]

7. Warntjes, M.; Engström, M.; Tisell, A.; Lundberg, P. Modeling the Presence of Myelin and Edema in the Brain Based on Multi-Parametric Quantitative MRI. Front. Neurol. 2016, 7, 16. [CrossRef]

8. Warntjes, J.B.M.; Leinhard, O.D.; West, J.; Lundberg, P. Rapid magnetic resonance quantification on the brain: Optimization for clinical usage. Magn. Reson. Med. 2008, 60, 320-329. [CrossRef]

9. Hagiwara, A.; Warntjes, M.; Hori, M.; Andica, C.; Nakazawa, M.; Kumamaru, K.K.; Abe, O.; Aoki, S. SyMRI of the Brain: Rapid Quantification of Relaxation Rates and Proton Density, With Synthetic MRI, Automatic Brain Segmentation and Myelin Measurement. Investig. Radiol. 2017, 52, 647-657. [CrossRef]

10. Hagiwara, A.; Hori, M.; Yokoyama, K.; Takemura, M.Y.; Andica, C.; Tabata, T.; Kamagata, K.; Suzuki, M.; Kumamaru, K.K.; Nakazawa, M.; et al. Synthetic MRI in the Detection of Multiple Sclerosis Plaques. Am. J. Neuroradiol. 2017, 38, 257-263. [CrossRef]

11. Warntjes, J.B.M.; Persson, A.; Berge, J.; Zech, W. Myelin Detection Using Rapid Quantitative MR Imaging Correlated to Macroscopically Registered Luxol Fast Blue-Stained Brain Specimens. Am. J. Neuroradiol. 2017, 38, 1096-1102. [CrossRef] [PubMed]

12. Ouellette, R.; Warntjes, M.; Forslin, Y.; Plattén, M.; Uppman, M.; Bergendal, Å.; Piehl, F.; Fredrikson, S.; Kristoffersen-Wiberg, M.; Mainero, C.; et al. Rapid estimation of myelin for diagnostic imaging (REMyDI)- A clinical and histopathological validation in multiple sclerosis. In Proceedings of the 26th Annual Meeting of ISMRM, Paris, France, 16-21 June 2018.

13. Hagiwara, A.; Hori, M.; Kamagata, K.; Warntjes, M.; Matsuyoshi, D.; Nakazawa, M.; Ueda, R.; Andica, C.; Koshino, S.; Maekawa, T.; et al. Myelin Measurement: Comparison Between Simultaneous Tissue Relaxometry, Magnetization Transfer Saturation Index and T1w/T2w Ratio Methods. Sci. Rep. 2018, 8, 1-12. [CrossRef] [PubMed]

14. Andica, C.; Hagiwara, A.; Hori, M.; Nakazawa, M.; Goto, M.; Koshino, S.; Kamagata, K.; Kumamaru, K.K.; Aoki, S. Automated brain tissue and myelin volumetry based on quantitative MR imaging with various in-plane resolutions. J. Neuroradiol. 2018, 45, 164-168. [CrossRef] [PubMed]

15. Fujita, S.; Hagiwara, A.; Hori, M.; Warntjes, M.; Kamagata, K.; Fukunaga, I.; Andica, C.; Maekawa, T.; Irie, R.; Takemura, M.; et al. Three-dimensional High-resolution Simultaneous Quantitative Mapping of the Whole Brain with 3D-QALAS: An Accuracy and Repeatability Study. Magn. Reson. Imaging 2019, 63, 235-243. [CrossRef] [PubMed]

16. Saccenti, L.; Andica, C.; Hagiwara, A.; Yokoyama, K.; Takemura, M.Y.; Fujita, S.; Maekawa, T.; Kamagata, K.; Le Berre, A.; Hori, M.; et al. Brain tissue and myelin volumetric analysis in multiple sclerosis at 3T MRI with various in-plane resolutions using synthetic MRI. Neuroradiology 2019, 61, 1219-1227. [CrossRef]

17. Hagiwara, A.; Hori, M.; Cohen-Adad, J.; Nakazawa, M.; Suzuki, Y.; Kasahara, A.; Horita, M.; Haruyama, T.; Andica, C.; Maekawa, T.; et al. Linearity, Bias, Intrascanner Repeatability and Interscanner Reproducibility of Quantitative Multidynamic Multiecho Sequence for Rapid Simultaneous Relaxometry at 3 T: A Validation Study With a Standardized Phantom and Healthy Controls. Investig. Radiol. 2019, 54, 39-47. [CrossRef]

18. Hagiwara, A.; Kamagata, K.; Shimoji, K.; Yokoyama, K.; Andica, C.; Hori, M.; Fujita, S.; Maekawa, T.; Irie, R.; Akashi, T.; et al. White Matter Abnormalities in Multiple Sclerosis Evaluated by Quantitative Synthetic MRI, Diffusion Tensor Imaging and Neurite Orientation Dispersion and Density Imaging. Am. J. Neuroradiol. 2019, 40, 1642-1648. [CrossRef]

19. Hagiwara, A.; Hori, M.; Yokoyama, K.; Nakazawa, M.; Ueda, R.; Horita, M.; Andica, C.; Abe, O.; Aoki, S. Analysis of White Matter Damage in Patients with Multiple Sclerosis via a Novel In Vivo MR Method for Measuring Myelin, Axons and G-Ratio. Am. J. Neuroradiol. 2017, 38, 1934-1940. [CrossRef]

20. Andica, C.; Hagiwara, A.; Hori, M.; Haruyama, T.; Fujita, S.; Maekawa, T.; Kamagata, K.; Yoshida, M.; Suzuki, M.; Sugano, H.; et al. Aberrant myelination in patients with Sturge-Weber syndrome analyzed using synthetic quantitative magnetic resonance imaging. Neuroradiology 2019, 61, 1387-1395. [CrossRef]

21. Andica, C.; Hagiwara, A.; Nakazawa, M.; Tsuruta, K.; Takano, N.; Hori, M.; Suzuki, H.; Sugano, H.; Arai, H.; Aoki, S. The Advantage of Synthetic MRI for the Visualization of Early White Matter Change in an Infant with Sturge-Weber Syndrome. Magn. Reson. Med. Sci. 2016, 15, 347-348. [CrossRef] 
22. Wallaert, L.; Hagiwara, A.; Andica, C.; Hori, M.; Yamashiro, K.; Koshino, S.; Maekawa, T.; Kamagata, K.; Aoki, S. The Advantage of Synthetic MRI for the Visualization of Anterior Temporal Pole Lesions on Double Inversion Recovery (DIR), Phase-sensitive Inversion Recovery (PSIR) and Myelin Images in a Patient with CADASIL. Magn. Reson. Med. Sci. 2018, 17, 275-276. [CrossRef] [PubMed]

23. Schmierer, K.; Scaravilli, F.; Altmann, D.R.; Barker, G.J.; Miller, D.H. Magnetization transfer ratio and myelin in postmortem multiple sclerosis brain. Ann. Neurol. 2004, 56, 407-415. [CrossRef] [PubMed]

24. Gass, A.; Barker, G.; Kidd, D.; Thorpe, J.W.; Macmanus, D.; Brennan, A.; Tofts, P.; Thompson, E.; McDonald, W.I.; Miller, D.H. Correlation of magnetization transfer ratio with clinical disability in multiple sclerosis. Ann. Neurol. 1994, 36, 62-67. [CrossRef] [PubMed]

25. Vavasour, I.M.; Laule, C.; Li, D.K.B.; Traboulsee, A.L.; MacKay, A.L. Is the magnetization transfer ratio a marker for myelin in multiple sclerosis? J. Magn. Reson. Imaging 2011, 33, 710-718. [CrossRef] [PubMed]

26. Henkelman, R.M.; Stanisz, G.J.; Graham, S.J. Magnetization transfer in MRI: A review. NMR Biomed. 2001, 14, 57-64. [CrossRef] [PubMed]

27. Helms, G.; Dathe, H.; Kallenberg, K.; Dechent, P. High-resolution maps of magnetization transfer with inherent correction for RF inhomogeneity and T1 relaxation obtained from 3D FLASH MRI. Magn. Reson. Med. 2008, 60, 1396-1407. [CrossRef]

28. Lema, A.; Bishop, C.; Malik, O.; Mattoscio, M.; Ali, R.; Nicholas, R.; Muraro, P.A.; Matthews, P.M.; Waldman, A.D.; Newbould, R.D. A Comparison of Magnetization Transfer Methods to Assess Brain and Cervical Cord Microstructure in Multiple Sclerosis. J. Neuroimaging 2017, 27, 221-226. [CrossRef]

29. Shafee, R.; Buckner, R.L.; Fischl, B. Gray matter myelination of 1555 human brains using partial volume corrected MRI images. NeuroImage 2015, 105, 473-485. [CrossRef]

30. Glasser, M.F.; Essen, D.C.V. Mapping Human Cortical Areas In Vivo Based on Myelin Content as Revealed by T1- and T2-Weighted MRI. J. Neurosci. 2011, 31, 11597-11616. [CrossRef]

31. Grydeland, H.; Walhovd, K.B.; Tamnes, C.K.; Westlye, L.T.; Fjell, A.M. Intracortical Myelin Links with Performance Variability across the Human Lifespan: Results from T1- and T2-Weighted MRI Myelin Mapping and Diffusion Tensor Imaging. J. Neurosci. 2013, 33, 18618-18630. [CrossRef]

32. Soun, J.E.; Liu, M.Z.; Cauley, K.A.; Grinband, J. Evaluation of neonatal brain myelination using the T1- and T2-weighted MRI ratio. J. Magn. Reson. Imaging 2017, 46, 690-696. [CrossRef] [PubMed]

33. Arshad, M.; Stanley, J.A.; Raz, N. Test-retest reliability and concurrent validity of in vivo myelin content indices: Myelin water fraction and calibrated T1w/T2w image ratio. Hum. Brain Mapp. 2017, 38, 1780-1790. [CrossRef]

34. Righart, R.; Biberacher, V.; Jonkman, L.E.; Klaver, R.; Schmidt, P.; Buck, D.; Berthele, A.; Kirschke, J.S.; Zimmer, C.; Hemmer, B.; et al. Cortical pathology in multiple sclerosis detected by the T1/T2-weighted ratio from routine magnetic resonance imaging. Ann. Neurol. 2017, 82, 519-529. [CrossRef] [PubMed]

35. Winklewski, P.J.; Sabisz, A.; Naumczyk, P.; Jodzio, K.; Szurowska, E.; Szarmach, A. Understanding the Physiopathology Behind Axial and Radial Diffusivity Changes-What Do We Know? Front. Neurol. 2018, 9 , 92. [CrossRef] [PubMed]

36. Song, S.-K.; Yoshino, J.; Le, T.Q.; Lin, S.-J.; Sun, S.-W.; Cross, A.H.; Armstrong, R.C. Demyelination increases radial diffusivity in corpus callosum of mouse brain. NeuroImage 2005, 26, 132-140. [CrossRef] [PubMed]

37. Klawiter, E.C.; Schmidt, R.E.; Trinkaus, K.; Liang, H.-F.; Budde, M.D.; Naismith, R.T.; Song, S.-K.; Cross, A.H.; Benzinger, T.L. Radial diffusivity predicts demyelination in ex vivo multiple sclerosis spinal cords. NeuroImage 2011, 55, 1454-1460. [CrossRef] [PubMed]

38. Campbell, J.S.W.; Leppert, I.R.; Narayanan, S.; Boudreau, M.; Duval, T.; Cohen-Adad, J.; Pike, G.B.; Stikov, N. Promise and pitfalls of g-ratio estimation with MRI. NeuroImage 2018, 182, 80-96. [CrossRef] [PubMed]

39. Lipp, I.; Jones, D.K.; Bells, S.; Sgarlata, E.; Foster, C.; Stickland, R.; Davidson, A.E.; Tallantyre, E.C.; Robertson, N.P.; Wise, R.G.; et al. Comparing MRI metrics to quantify white matter microstructural damage in multiple sclerosis. Hum. Brain Mapp. 2019, 40, 2917-2932. [CrossRef] [PubMed]

40. Polman, C.H.; Reingold, S.C.; Banwell, B.; Clanet, M.; Cohen, J.A.; Filippi, M.; Fujihara, K.; Havrdova, E.; Hutchinson, M.; Kappos, L.; et al. Diagnostic criteria for multiple sclerosis: 2010 Revisions to the McDonald criteria. Ann. Neurol. 2011, 69, 292-302. [CrossRef] [PubMed]

41. Ganzetti, M.; Wenderoth, N.; Mantini, D. Whole brain myelin mapping using T1- and T2-weighted MR imaging data. Front. Hum. Neurosci. 2014, 8, 671. [CrossRef] 
42. Andersson, J.; Sotiropoulos, S. An integrated approach to correction for off-resonance effects and subject movement in diffusion MR imaging. NeuroImage 2015, 125, 1063-1078. [CrossRef] [PubMed]

43. Hagiwara, A.; Hori, M.; Yokoyama, K.; Takemura, M.Y.; Andica, C.; Kumamaru, K.K.; Nakazawa, M.; Takano, N.; Kawasaki, H.; Sato, S.; et al. Utility of a Multiparametric Quantitative MRI Model That Assesses Myelin and Edema for Evaluating Plaques, Periplaque White Matter and Normal-Appearing White Matter in Patients with Multiple Sclerosis: A Feasibility Study. Am. J. Neuroradiol. 2017, 38, 237-242. [CrossRef] [PubMed]

44. Guo, A.C.; MacFall, J.R.; Provenzale, J.M. Multiple Sclerosis: Diffusion Tensor MR Imaging for Evaluation of Normal-appearing White Matter. Radiology 2002, 222, 729-736. [CrossRef] [PubMed]

45. Wilcox, R.R. Comparing dependent robust correlations. Br. J. Math. Stat. Psychol. 2016, 69, $215-224$. [CrossRef] [PubMed]

46. comp2dcorr. Available online: https://github.com/GRousselet/blog/tree/master/comp2dcorr (accessed on 15 December 2019).

47. Benjamini, Y.; Krieger, A.M.; Yekutieli, D. Adaptive linear step-up procedures that control the false discovery rate. Biometrika. 2006, 93, 491-507. [CrossRef]

48. Dziedzic, T.; Metz, I.; Dallenga, T.; König, F.B.; Müller, S.; Stadelmann, C.; Brück, W. Wallerian degeneration: A major component of early axonal pathology in multiple sclerosis. Brain Pathol. 2010, 20, 976-985. [CrossRef] [PubMed]

49. Moll, N.M.; Rietsch, A.M.; Thomas, S.; Ransohoff, A.J.; Lee, J.-C.; Fox, R.; Chang, A.; Ransohoff, R.M.; Fisher, E. Multiple Sclerosis Normal-Appearing White Matter: Pathology-Imaging Correlations. Ann. Neurol. 2011, 70, 764-773. [CrossRef] [PubMed]

50. Kessler, L.G.; Barnhart, H.X.; Buckler, A.J.; Choudhury, K.R.; Kondratovich, M.V.; Toledano, A.; Guimaraes, A.R.; Filice, R.; Zhang, Z.; Sullivan, D.C. The emerging science of quantitative imaging biomarkers terminology and definitions for scientific studies and regulatory submissions. Stat. Methods Med. Res. 2015, 24, 9-26. [CrossRef]

51. Bells, S.; Morris, D.; Vidarsson, L. Comparison of Linear Combination Filtering to DTI and MTR in Whole Brain Myelin-Water Imaging. In Proceedings of the 15th Annual Meeting of ISMRM, Berlin, Germany, 19-25 May 2007; Abstract 1606.

52. O'Muircheartaigh, J.; Vavasour, I.; Ljungberg, E.; Li, D.K.B.; Rauscher, A.; Levesque, V.; Garren, H.; Clayton, D.; Tam, R.; Traboulsee, A.; et al. Quantitative neuroimaging measures of myelin in the healthy brain and in multiple sclerosis. Hum. Brain Mapp. 2019, 40, 2104-2116. [CrossRef] [PubMed]

53. Uddin, M.N.; Figley, T.D.; Solar, K.G.; Shatil, A.S.; Figley, C.R. Comparisons between multi-component myelin water fraction, $\mathrm{T} 1 \mathrm{w} / \mathrm{T} 2 \mathrm{w}$ ratio and diffusion tensor imaging measures in healthy human brain structures. Sci. Rep. 2019, 9, 2500. [CrossRef]

54. Ludwin, S.K. The Pathogenesis of Multiple Sclerosis: Relating Human Pathology to Experimental Studies. J. Neuropathol. Exp. Neurol. 2006, 65, 305-318. [CrossRef] [PubMed]

55. Barkhof, F. The clinico-radiological paradox in multiple sclerosis revisited. Curr. Opin. Neurol. 2002, 15, 239-245. [CrossRef] [PubMed]

56. Obberghen, E.V.; Mchinda, S.; Le Troter, A.; Prevost, V.H.; Viout, P.; Guye, M.; Varma, G.; Alsop, D.C.; Ranjeva, J.-P.; Pelletier, J.; et al. Evaluation of the Sensitivity of Inhomogeneous Magnetization Transfer (ihMT) MRI for Multiple Sclerosis. Am. J. Neuroradiol. 2018, 39, 634-641. [CrossRef] [PubMed]

57. Blystad, I.; Håkansson, I.; Tisell, A.; Ernerudh, J.; Smedby, Ö.; Lundberg, P.; Larsson, E.-M. Quantitative MRI for Analysis of Active Multiple Sclerosis Lesions without Gadolinium-Based Contrast Agent. AJNR Am. J. Neuroradiol. 2016, 37, 94-100. [CrossRef]

(C) 2020 by the authors. Licensee MDPI, Basel, Switzerland. This article is an open access article distributed under the terms and conditions of the Creative Commons Attribution (CC BY) license (http://creativecommons.org/licenses/by/4.0/). 